Folia Toruniensia - 19/2019

\title{
Janusz Bonczkowski
}

Staatsarchiv in Thorn, Polen

e-mail: jbonczkowski@torun.ap.gov.pl

ORCID ID: 0000-0003-1033-2802

\section{riedrich Julius Kleinmichel - Genremaler und Illustrator (1846-1892)}

DOI: http://dx.doi.org/10.12775/FT.2018.012

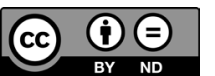

Dieser Text wird unter der Creative Commons-Lizenz Namensnennung-Keine Bearbeitungen 4.0 International (CC BY-ND 4.0) veröffentlicht.

Dr. Janusz Bonczkowski ist Mitarbeiter des Staatsarchivs in Thorn, Absolvent des Geschichtsstudiums (Archivkunde) an der Fakultät für Geschichtswissenschaften der NikolausKopernikus-Universität in Thorn sowie des Aufbaustudiums im Bereich der Programmierung und Anwendung von Computern. 2017 promovierte er in den Geisteswissenschaften mit seiner Dissertation: „Analiza dyplomatyczno-źródłoznawcza księgi czynszowej miasta Grudziądza z lat 1502-1516 wraz z jej edycją źródłową“ („Diplomatische Quellenanalyse und Edition des Zinsbuches der Stadt Graudenz aus den Jahren 1502-1516“). In sein Interessenfeld rückten die Stadtgeschichte Graudenz' und anderer kleinerer Städte des Königlichen Preußen vom Mittelalter bis zur Neuzeit sowie die Quellenedition. Er ist Mitglied des Vereins der Polnischer Archivare und der Wissenschaftlichen Gesellschaft zu Thorn.

tichworte: Friedrich Julius Kleinmichel; Julius Lohmeyer; Rosen/Rodzone; Königsberg; München; Kunstakademie in Königsberg

usammenfassung. These/Ziel: Der vorliegende Beitrag bezieht sich auf die Gestalt Friedrich Julius Kleinmichels, eines aus Rosen/Rodzone bei Löbau gebürtigen Genremalers, Buch- und Zeitschriftenillustrators. Forschungsmethode: Um die Person und das Schaffen Friedrich Julius Kleinmichels darzustellen, untersuchte man die zugängliche Literatur, die Zeitungen aus dem 19. Jahrhundert, Bücher mit seinen Bildern sowie Ausstellungskataloge und Auktionen seiner Gemälde. Ergebnisse/Schlussfolgerungen: Die Themenvielfalt der Gemälde, Buch- und Zeitschriftenillustrationen Friedrich Julius Kleinmichels ist bezogen vor allem auf die Kinderwelt. Er arbeitete zusammen vor allem mit den Schriftstellern und Dichtern der Kinderliteratur wie etwa Victor Blüthgen, Helen Binder, Emma Biller, Julius Lohmeyer, Johannes Trojan, Franz Dittmar.

Die zweite Hälfte des 19. Jahrhunderts brachte den Umbruch im Buchillustrationsstil. Das Bild dominierte den Text. Nach Ansätzen des neuen Stils sollte man in England suchen, wo das sog. toy-book erschienen ist. Unter die Vordenker dieses neuen Trends zählt man Edmund Evans, Walter Crane, Randolph Caldecott, 


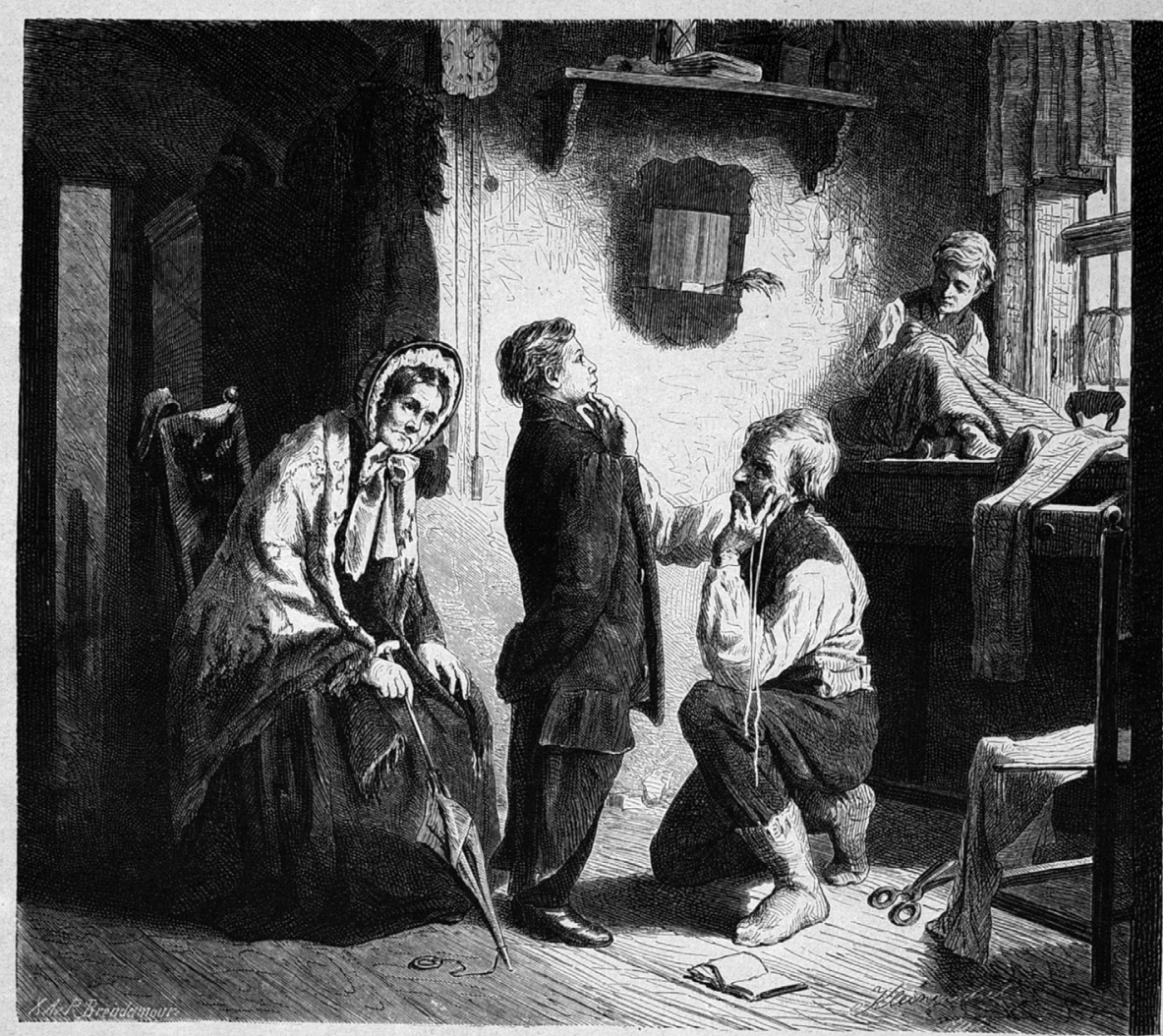

Bom (ङrujbater auf ben Enter.

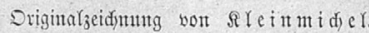

Abb. 1. J. Kleinmichel, Vom Großvater auf den Enkel

Quelle: Die Gartenlaube, Nr. 32, Leipzig 1872, S. 519.

Kate Greenaway, Elsa Beskow und Wiliam Morris. Die in die Bücher eingefügte Bilder knüpften an die Renaissancezeit an, und zu jener Zeit, als die Art Nouveau, der Modernismus und der Jugendstil präsent waren, auch an die Volkskunst und -symbolik. Die Buchillustrationen enthielten zahlreiche Pflanzenelemente sowie märchenhafte und magische Motive. Einer der Vertreter dieser Richtung der Buchund Zeitschriftenillustrierung in Deutschland war Friedrich Julius Kleinmichel' ${ }^{1}$. Er wurde am 5. März 1846 als Sohn eines Oberförsters im Dorf Rosen (poln. Rodzone) bei Löbau geboren ${ }^{2}$. Nach dem vorzeitigen Tod seiner Eltern wurde der kleine Julius in Obhut der Verwandten aus Königsberg gegeben, die inn anschließend adoptierten. Dem Wunsch der Pflegeeltern zufolge sollte er technische Ausbildung genießen, und später, nach dem Lehrabschluss, ihr Geschäft übernehmen. Sehr

E. Zarych, Ilustracje dla dzieci, [in:] Encyklopedia dzieciństwa [online], [Zugang 27 VI 2019]. Zugänglich auch bei World Wide Web: http://encyklopediadziecinstwa.pl/index.php/llustracje_dla_dzieci; J. Wiercińska, Sztuka i Książka, Państwowe Wydawnictwo Naukowe, Warszawa 1986, S. 89; D. Bland, A History of Book Illustration, London 1958, S. $255,268,270$.

2 In der Fachliteratur wird Rodzonne bei Graudenz als sein Geburtsort erwähnt; F. Boetticher, Malerwerke des neunzehnten Jahrhunderts. Beitrag zur Kunstgeschichte, Bd. 1, Dresden 1891, S. 698; H. Holland, Kleinmichel, Fr. Julius, [in:] Deutsche Biographie - Onlinefassung [online], [Zugang 5 I 2019]. Zugänglich bei World Wide Web: http://www.deutsche-biographie.de/sfz42614.html. 
schnell erwies sich aber, dass Friedrich Julius künstlich begabt war und immer mehr Stunden mit Zeichnen verbrachte. Die Kunstlehre begann er in der Königsberger Kunstschule bei Karl Ludwig Rosenfelder ${ }^{3}$. Sein erstes Werk stellte er 1870 zur Schau. Für seine humoristische Illustration „Vom Großvater auf den Enkel vererbten Frack" ${ }^{4}$ wurde er positiv bewertet, was ihn ermunterte, weiterhin daran zu arbeiten ${ }^{5}$.

Nach dem Tode der Pflegeeltern 1871 reiste er nach Berlin, um sich weiter bilden zu lassen. Seine Bilder, die von der Reise nach Rügen inspiriert wurden, veröffentlichte er 1872 in der Zeitschrift „Der Bazar Illustrierte Damen-Zeitung“, darunter beispielsweise die Szene „Vor dem Pfarrhause“ oder „Von der Insel Rugen“6. In dieser Zeitspanne lernte er Johann Arthur Severin Nikutowski kennen ${ }^{7}$, der inn nach Düsseldorf einlud, wo er anschließend die Ausbildung in der Bildkunst an der KöniglichPreußischen Kunstakademie fortsetzte. Seine Werke, darunter „Fischer-Scenen“, „Konfirmandinnen vor der Kirche“ und „eine Vogelscheuche plündernde Vagabund“, publizierte er in der „Allgemeinen Frauen-Zeitung“". Trotz gesundheitlicher Probleme, die inn damals plagten, betätigte sich Kleinmichel vornehmlich als Illustrator für verschiedene Zeitschriften, wie etwa für das „Daheim“ in Leipzig, das 1878 inm die Stelle als artistischer Leiter übertrug, oder die „Deutsche Jugend“. Zu diesem Zeitpunkt heiratete er Selma Dunsky aus Tiflis und befreundete sich mit Julius Lohmeyer ${ }^{9}$. 1882 zog er nach München, wo er an der Schellingsstraße 114 seine Werkstatt eröffnete ${ }^{10}$.

Die Arbeit für die Zeitschriften, die vor allem für die Kinder und Jugendlichen vorgesehen waren, gestaltete Kleinmichel als Künstler und wies ihm die Richtung und den Raum in der Kunst zu. Einen großen Einfluss auf sein Schaffen hatten die Arbeiten von Ludwig Richter ${ }^{11}$ sowie von der britischen Schriftstellerin und IIlustratorin Kate Greenaway ${ }^{12}$. In seinen Bildern, Grafiken und Gemälden schilderte er vor allem Kinder in verschiedenen Lebensmomenten. Es waren Szenen voll Freude sowie Schmerz, beim Spiel sowie in ganz seriösen Situationen. Ihm gelang es, die Kinderemotionen genau nachzubilden, und zwar sowohl beim Nachsinnen

3 Karl Ludwig Rosenfelder (1813-1881), deutscher Maler, spezialisiert auf historische Themen; v. Donop, Rosenfelder Karl Ludwig R., [in:] Deutsche Biographie - Onlinefassung [online], [Zugang 25 VII 2019]. Zugänglich bei World Wide Web: https://www.deutsche-biographie.de/sfz76967.html; H. Holland, op. cit. Zugänglich bei World Wide Web: http://www.deutsche-biographie.de/sfz42614.html.

4 Dieses Bild erschien später in der Zeitschrift: Die Gartenlaube, 1872, Nr. 32, S. 519.

5 F. Boetticher, op. cit., S. 698-699; H. Holland, op. cit., [Zugang 5 I 2019]. Zugänglich bei World Wide Web: http:// www.deutsche-biographie.de/sfz42614.html.

6 Der Bazar Illustrierte Damen-Zeitung, 1872, Nr. 16, S. 125; Ibidem, 1872, Nr. 34, S. 273.

7 Johann Arthur Severin Nikutowski (1830-1888), deutscher Maler, der in seinem Schaffen besonders historische Themen und Schlachtenmotive auswählte; H. A. Müller, Biographisches Künstler-Lexikon, Leipzig 1882, S. 394.

8 Allgemeine Frauen-Zeitung, 1873, Nr. 6, S. 44; H. Holland, op. cit., [Zugang 5 I 2019]. Zugänglich bei World Wide Web: http://www.deutsche-biographie.de/sfz42614.html.

9 Julius Lohmeyer (1835-1903), deutscher Dichter und Autor der Jugendliteratur; E. M. Brockhoff, Lohmeyer Julius, [in:] Neue Deutsche Biographie, Bd. 15, Berlin 1987, S. 133-134; A. Klotz, Kinder- und Jugendliteratur in Deutschland 1840-1950, Gesamtverzeichnis der Veröffentlichungen in deutscher Sprache, Bd. III L-Q, Stuttgart 1994, S. 80; F. Boetticher, op. cit., S. 698; H. Holland, op. cit., [Zugang 5 I 2019]. Zugänglich bei World Wide Web: http://www. deutsche-biographie.de/sfz42614.html.

10 Illustrierter Katalog der Münchener Jahresausstellung von Kunstwerken Aller Nationen im kgl. Glaspalaste 1891, 3. Aufl., ausgeg. am 24. Juli, München 1891, S. 54; Illustrierter Katalog der VI. Internationalen Kunst-Ausstellung $1892 \mathrm{im} \mathrm{Kgl.} \mathrm{Glaspalaste,} \mathrm{1.} \mathrm{Juni} \mathrm{-} \mathrm{Ende} \mathrm{Oktober,} \mathrm{2.} \mathrm{Aufl.,} \mathrm{ausgeg.} \mathrm{am} \mathrm{20.} \mathrm{Juni} \mathrm{1892,} \mathrm{München,} \mathrm{1892,} \mathrm{S.} 43$.

11 Ludwig Richter (1803-1884), deutscher Maler, Philosoph und Ästhet, einer der wichtigsten deutschen romantischen Philosophen; H. Börsch-Supanh, Richter Adrian Ludwig, [in:] Neue Deutsche Biographie, Bd. 21, Berlin 2003, S. 535-537.

12 Kate Greenaway (1846-1901), eine britische Schriftstellerin und Illustratorin, verbunden mit dem Londoner Kunstverein Pre-Raphaelite Brotherhood; Kate Greenaway (1846-1901), [in:] The Victorian Web, [online], [Zugang 1 I 2019]. Zugänglich bei World Wide Web: http://www.victorianweb.org/art/illustration/ greenaway/. 
als auch während ihrer Aktivitäten zu Hause, draußen oder am See. Er hielt die Szenen zutreffend, packend und humorvoll fest ${ }^{13}$.

Seine Bilder befinden sich im Gedichtsbuch Victos Blüthgens „Im Flügelkleide“, das in Berlin 1881 veröffentlicht wurde ${ }^{14}$. In den drei darauffolgenden Jahren erschienen Bücher mit seinen Illustrationen, d.i. „Kinderhort in Bild und Wort“ (1882) mit dem Text Helene Binders ${ }^{15}$, „Lieschens kluge Einfälle. Hänschens Reise durch die Stube. Mariele und sieben Puppen. Wie es Fritzchen auf seiner Schwester. Hochzeit Erging. Vier Erzählung“ (1882) mit dem Text Emmy Billers ${ }^{16}$ und „Kinderhumor“17 (1886) mit den Gedichten Julius Lohmeyers und Johannes Trojans $^{18}$, sowie in anderen Büchern ${ }^{19}$. Die Übersetzungen der Texte gewannen an Popularität auch im Ausland. Zu den wichtigsten Werken Kleinmichels gehören das Buch „Aus der Jugendzeit“ mit den Gedichten Franz Dittmars ${ }^{20}$, „Für kleine Leute“21 (1883) sowie „Die Welt vom Fenster aus“22 (1880) mit den Gedichten Johann Trojans, „Kinderwelt“23 (1886), „Mein Vaterhaus“24 (1887) und „Schnurrige Kerle“25 (1890). Außer den Kinderbüchern illustrierte Julius Kleinmichel auch Bücher für die ältere Lesergruppe ${ }^{26}$.

Erwähnenswert ist die einzige polnische Übersetzung des Buches Heinrich Hoffmans „Der Struwwelpeter“ mit den Abbildungen Kleinmichels, herausgegeben in Petersburg 1883 unter dem Titel „Obrazków świat dziecinnych lat: opowiadania wierszem autora ,Złotej rószczki‘ z rycinami kolorowanemi według akwareli J. Kleinmichela"27.

${ }^{13}$ Es bewährten sich keine Quellen zu den Reisen Julius Kleinmichels in seinen Geburtsort bzw. ins Kulmer Land. Es ist auch unbekannt, ob die Kindheit im Dorf Rosen / Rodzone irgendeinen Einfluss auf sein Schaffen hatte; $\mathrm{H}$. Holland, op. cit., [Zugang 5 I 2019]. Zugänglich bei World Wide Web: http://www.deutsche-biographie.de/sfz42614. html.

14 Victor Blüthgen (1844-1920), deutscher Schriftsteller und Dichter; F. Bochow, Blüthgen Victor August Eberhard, [in:] Sächsische Biografie, [online] [Zugang 1 I 2019]. Zugänglich bei World Wide Web: http://saebi.isgv.de/ biografie/Victor_BI\%C3\%BCthgen_(1844-1920); J. Kleinmichel, V. Blüthgen, Im Flügelkleide. Bilder \& Reime aus der Kinderwelt, Berlin 1881.

15 Helene Binder (1855-1915), deutsche Schriftstellerin; A. Klotz, op. cit., Bd. I A-F, Stuttgart 1990, S. 153; H. Binder, J. Kleinmichel, Kinderhort in Bild und Wort, München 1883.

${ }^{16}$ Emma Wuttke-Biller (1833-1933), deutsche Schriftstellerin; A. Klotz, op. cit., Bd. I A-F, Stuttgart 1990, S. 151; E. Biller, Lieschens kluge Einfälle. Hänschens Reise durch die Stube. Mariele und sieben Puppen. Wie es Fritzchen auf seiner Schwester. Hochzeit Erging. Vier Erzählung, München 1882.

17 J. Lohmeyer, J. Trojan, J. Kleinmichel, Kinderhumor, Leipzig 1886.

18 Trojan Johannes (1835-1903), deutscher Schriftsteller und Dichter. Klotz, A., op. cit., Bd. V T-Z, Stuttgart 1999, s. 51.

19 B. Augusti, Haus und Welt. Bilder aus des Lebens Mai, eine Fortsetzung der Mädchenlose. Ihren Jungen Freundingen gewidmet, Leipzig 1883; Idem, Knospen und Blütten für junge Mädchen, Leipzig 1884; J. Lohmeyer, J. Kleinmichel, F. Schanz, Eine Prinzenreise. Mitte heiteren Reimen von Frida Schanz und Julius Lohmeyer, Leipzig und New York 1888; C. Jäger, Die Wunderflasche. Wie du mir, so ich dir. Zwei Schauspiele für die Jugend, Stuttgart 1881; G. Bötticher, J. Kleinmichel, Herrn Dietchens Erzählungen und andere Dialekthumoresken, Leipzig 1890; G. Bötticher, Schilda. Verse eines Kleinstädters, Leipzig 1898; F. Bonn, Der Weber-Hannes. Durchgebrannt! Zwei Erzählungen, Stuttgart 1880; U. B. Celius, Aus dem Zauberland! Alte Märchen, Leipzig 1882; Ch. A. Murray, Prärievogel, StuttgartBerlin-Leipzig 1890; E. Bormann, Das Buch von der Mutter Natur, Leipzig 1888; Ibidem, Das Buch der Bücherwelt, Leipzig 1888; F. Hoffmann, Die Großmutter im Kreise ihrer Enkel, Stuttgart 1877; J. Ringelnatz, W. Pape, Parodie und Selbstparodie in Leben und Werk, Berlin und New York 1974, S. 356-357.

${ }^{20}$ Franz Ditmar (1857-1915), deutscher Schriftsteller; A. Klotz, op. cit., Bd. I A-F, Stuttgart 1990, S. 343; F. Diettmar J. Kleinmichel, Aus der Jugendzeit. Geschichte für die Kinderwelt, Leipzig 1886.

21 L. Kramer, Für kleine Leute. Bilder und Reime, München 1883.

22 J. Trojan, J. Kleinmichel, Die Welt vom Fenster aus, Breslau 1880.

23 J. Kleinmichel, C. Röhling, Kinderwelt. 230 Lieder, Sprüche und Reime, Berlin 1886.

${ }^{24}$ J. Kleinmichel, J. Lohmeyer, Mein Vaterhaus. Ein heiteres Kindertagebuch, Leipzig 1887.

25 G. Bötticher, Schnurrige Kerle, Leipzig 1890.

${ }^{26}$ E. Marlitt, Reichsgräfin Gisela, Leipzig 1899.

27 W. Szymanowski, Obrazków świat z dziecinnych lat: opowiadania wierszem autora „Złotej rószczki“ z rycinami kolorowanemi według akwarelli J. Kleinmichla, Petersburg 1883. 


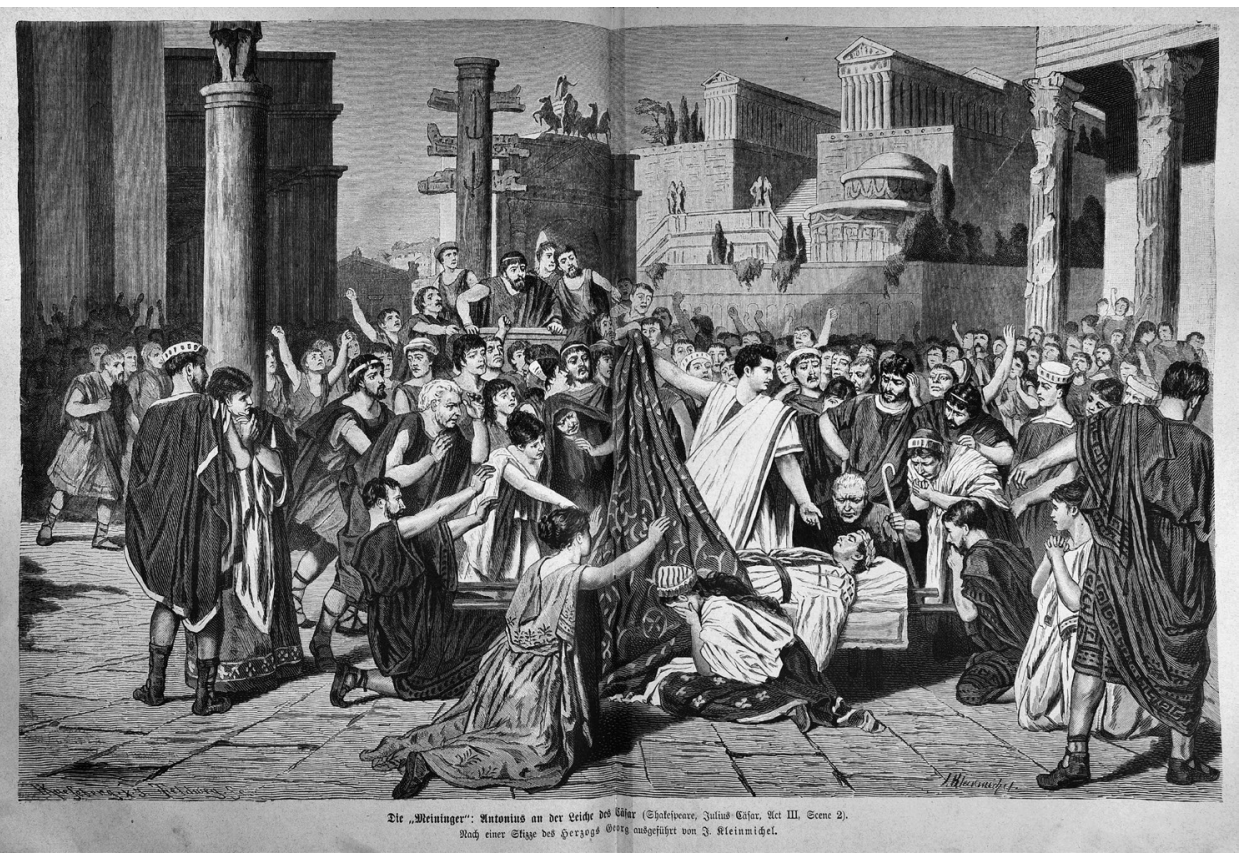

Abb. 2. J. Kleinmichel, Antonius an der Leiche des Cesar

Quelle: Die Gartenlaube, Nr. 14, Leipzig 1879, S. 236-237.

Zur selben Zeit, als er die Bilder für Bücher zeichnete, veröffentlichte er seine Arbeiten auch in den Zeitschriften, darunter in "Der Gartenlaube“ und den „Fliegenden Blättern“28, und malte für den Künstlerverein in München sowie für die lokalen großen Ausstellungen im Glaspalast ${ }^{29}$, u.a. „Ein Kinderfest im Walde“, „Eifrigen Botaniker" (1884), „Schwierigen Anfang im Schreibunterricht“ (1887), „Ein Besuch“, „Eine Entführung“ (1890), „Ein Nimmersatt“ (1891), „Wichtiger Brief“ $(1892)^{30}$. Während seiner letzten Reise arbeitete er in Rügen, Tirol und Capri, wo er zahlreiche Themen für die künftigen Gemälde fand. In Bozen erlitt er einen klei-

${ }_{28}$ Beispielsweise Fliegende Blätter, 1889, Nr. 2284, S. 163; Ibidem, 1891, Nr. 2378, S. 71; Ibidem, 1891, Nr. 2418, S. 196; Ibidem, 1892, Nr. 2433, S. 93; Die Gartenlaube, Nr. 14, Leipzig 1879, S. 236-237; Ibidem, 1883, Nr. 18, S. 297; Ibidem, 1889, Nr. 14, S. 441; Ibidem, 1890, Nr. 7, S. 213.

29 Ausstellungspalast in München 1854-1931, in seiner Form basierend auf Crystal Palace in London; R. Bauer, E. Piper, München. Geschichte einer Stadt, München 1993, S. 179.

30 Auf den Webseiten stößt man zudem auf andere Bilder dieses Künstlers wie etwa auf „Kleines Mädchen mit Zahnschmerzen“, „Sonntagsnachmittag an der Grafenberger Chaussee“, „Alte Frau In Der Kapelle“, „Spielende Kinder“, „Der erste Schritt“; Julius Kleinmichel, [in:] Invaluable [online], [Zugang 5 I 2019]. Zugänglich bei World Wide Web: https://www.invaluable.com/auction-lot/1846-rodzonne-1892-munichsunday-afternoon-on-th-682-c-9d 545dbbf8; Julius Kleinmichel, [in:] Bukowskis - Arts \& business [online], [Zugang 5 I 2019]. Zugänglich bei World Wide Web:www.bukowskis.com/en/lots/797903-julius-kleinmichel-julius-kleinmichel-oil-on-canvas-signed-and -datedleipzig-78; Ferdinand Julius Theodor Kleinmichel, [in:] Blouin Art [online], [Zugang 5 I 2019]. Zugänglich bei World Wide Web: https://blouinartsalesindex.com/auctions/Ferdinand-Julius-Theodor-Kleinmichel-4927072/Playtime-; Ferdinand Julius Theodor Kleinmichel, [in:] Blouin Art [online], [Zugang 5 I 2019]. Zugänglich bei World Wide Web: https://blouinartsalesindex.com/auctions/Ferdinand-Julius-Theodor-Kleinmichel-4993665/Alte-Frau-in-der-Kapelle-; Ferdinand Julius Theodor Kleinmichel, [w:] Blouin Art [online], [Zugang 5 I 2019]. Zugänglich bei World Wide Web: https://blouinartsalesindex.com/auctions/Ferdinand-Julius-Theodor-Kleinmichel-1084228/The-first-step-1883; F. Boetticher, op. cit., S. 698-699; Illustrierter Katalog der Münchener Jahresausstellung von Kunstwerken Aller Nationen im königl. Glaspalaste 1889, 1. Aufl., ausgegeben am 1. Juli, München 1889, S. 49; Illustrierter Katalog der Münchener Jahresausstellung von Kunstwerken Aller Nationen im königl. Glaspalaste 1890, ausgeg. Anfang September, München 1890, S. 20; Illustrierter Katalog der Münchener Jahresausstellung von Kunstwerken Aller Nationen im kgl. Glaspalaste 1891, 3. Aufl., ausgeg. am 24. Juli, München 1891, S. 54, 182; Illustrierter Katalog der VI. Internationalen Kunst-Ausstellung $1892 \mathrm{im} \mathrm{Kgl.} \mathrm{Glaspalaste,} \mathrm{1.} \mathrm{Juni} \mathrm{-} \mathrm{Ende} \mathrm{Oktober,} \mathrm{2.} \mathrm{Aufl.,} \mathrm{ausgeg.} \mathrm{am}$ 20. Juni 1892, München 1892, S. 43. 
nen Hitzschlag. Als er wieder genas und nach München zurückkehrte, erkrankte er an einer akuten Bronchitis, infolge deren er am 12. August 1892 im Alter von 46 Jahren verstarb ${ }^{31}$.

Wenn man das Schaffen des aus einem Dorf in der Nähe von Löbau gebürtigen Julius Kleinmichel zusammenfassen will, soll man darauf hinweisen, dass er zu einem kleinen Kreis der hervorragenden Buch- und Zeitungsillustratoren sowie Maler gehörte, deren Arbeiten vor allem auf Kinderfragen bezogen waren. Er arbeitete mit vielen deutschen Kinderschriftstellern der zweiten Hälfte des 19. Jahrhunderts zusammen, darunter Julius Lohmeyer, Victor Blüthgen, Helen Binder, Emma Biller, Johannes Trojan und Franz Dittmar. Seine Bilder erschienen in Neuauflagen der oben genannten Publikationen sogar viele Jahre nach seinem Tode. 2017 erinnerte man an das Schaffen Julius Kleinmichels und veröffentlichte erneut sein 1886 herausgegebenes Buch „Kinderwelt - 230 Lieder, Sprüche und Reime: Mit 50 Bildern von Julius Kleinmichel und Carl Röhling“32.

\section{Bibliographie}

Kleinmichel, Julius. 1873. „Konfirmandinnen vor der Kirche“, „eine Vogelscheuche plündernde Vagabund“. Allgemeine Frauen-Zeitung, 6: 44.

Kleinmichel Julius. 1872. „Vom Großvater auf den Enkel”. Die Gartenlaube, 32: 519 .

Kleinmichel, Julius. 1879. „Antonius an der Leiche des Cäsar". Die Gartenlaube 14: 236-237.

Kleinmichel, Julius. 1883. [ilustracja przedstawiająca siedzącą kobietę przy kołysce zmarłego dziecka] Die Gartenlaube 18: 297.

Kleinmichel, Julius. 1889. „Großmutters Geburtstag“ Die Gartenlaube 14:441.

Kleinmichel, Julius. 1890. „Der Besuch“ Die Gartenlaube 7: 213.

Kleinmichel, Julius. 1872. „Vor dem Pfarrhause“ Der Bazar Illustrierte Damen-Zeitung 16: 125.

Kleinmichel, Julius. 1872. „Von der Insel Rugen“. Der Bazar Illustrierte Damen-Zeitung 34: 273.

Kleinmichel, Julius. 1889. „Gedichte eines Kleinstädters“ Fliegende Blätter 2284: 163.

Kleinmichel, Julius. 1891. „Motivirung“. Fliegende Blätter 2378: 71.

Kleinmichel, Julius. 1891. „Bei der Schmmiere“. Fliegende Blätter 2418: 196.

Kleinmichel, Julius. 1892. „In gehobener Stimmung“. Fliegende Blätter 2433: 93.

NN. 1889. Illustrierter Katalog der Münchener Jahresausstellung von Kunstwerken Aller Nationen im königl. Glaspalaste 1889, 1. Aufl., ausgegeben am 1. Juli: Friedrich Bruckmann.

\footnotetext{
${ }^{31}$ F. Boetticher, op. cit., S. 698-699; H. Holland, op. cit., [Zugang 5 I 2019]. Zugänglich bei World Wide Web: http:// www.deutsche-biographie.de/sfz42614.html.

32 H. L. Koester, Geschichte der Deutschen Jugendliteratur in Monographien, Bd. 1, Hamburg 1906, S. 26-27; A. Wildermuth, Mein Bruder und ich, Stuttgart - Berlin - Leipzig 1898; B. Augusti, Haus und Welt. Bilder aus des Lebens Mai. Ihren jungen Freundinnen gewidmet, Leipzig 1902; C. Schmid, Hundert kurze Erzählungen, MünchenOldenbourg 1910; E. Bormann, Humoristischer Hausschatz, Berlin, 1921.
} 
NN. 1890. Illustrierter Katalog der Münchener Jahresausstellung von Kunstwerken Aller Nationen im königl. Glaspalaste 1890, ausgeg. Anfang September: Franz Hanfstaengl Kunstverlag.

NN. 1891. Illustrierter Katalog der Münchener Jahresausstellung von Kunstwerken Aller Nationen im kgl. Glaspalaste 1891, 3. Aufl., ausgeg. am 24. Juli: Friedrich Bruckmann.

NN. 1892. Illustrierter Katalog der VI. Internationalen Kunst-Ausstellung 1892 im Kgl. Glaspalaste, 1. Juni - Ende Oktober, 2. Aufl., ausgeg. am 20. Juni 1892: Friedrich Bruckmann.

Augusti, Brigitte. 1883. Haus und Welt. Bilder aus des Lebens Mai, eine Fortsetzung der Mädchenlose. Ihren Jungen Freundingen gewidmet. Leipzig: Ferdinand Hirt \& Sohn.

Augusti, Brigitte. 1902. Haus und Welt. Bilder aus des Lebens Mai. Ihren jungen Freundinnen gewidmet. Leipzig: Ferdinand Hirt \& Sohn.

Augusti, Brigitte. 1884. Knospen und Blütten für junge Mädchen. Leipzig: Ferdinand Hirt \& Sohn.

Bauer, Reinhard und Ernst Piper. 1993. München. Geschichte einer Stadt. München: Deutscher Taschenbuch-Verlag.

Biller, Emma. 1882. Lieschens kluge Einfälle. Hänschens Reise durch die Stube. Mariele und sieben Puppen. Wie es Fritzchen auf seiner Schwester. Hochzeit Erging. Vier Erzählung. München: Theo Stroefer's Kunstverlag.

Binder, Helene, Kleinmichel, Julius. 1883. Kinderhort in Bild und Wort. München: Theo Stroefer's Kunstverlag.

Bland, David. 1958. A History of Book Illustration. London: Faber and Faber Limited.

Boetticher, Friedrich. 1891. Malerwerke des neunzehnten Jahrhunderts. Beitrag zur Kunstgeschichte, 1. Dresden: Fr. v. Boetticher's Verlag.

Bochow, Fedor. 2004. „Blüthgen Victor August Eberhard”. W Sächsische Biografie. Dostęp Styczeń 1. http://saebi.isgv.de/biografie/Victor_Bl\%C3\%BCthgen_(1844-1920).

Bonn, Franz. 1880. Der Weber-Hannes. Durchgebrannt! Zwei Erzählungen. Stuttgart: Kröner.

Bormann, Edwin. 1888. Das Buch von der Mutter Natur. Leipzig: Bormann.

Bormann, Edwin. 1888. Das Buch der Bücherwelt. Leipzig: Bormann.

Bormann, Edwin. 1921. Humoristischer Hausschatz. Berlin: Bormann.

Börsch-Supanh, Helmut. 2003. „Richter Adrian Ludwig“. W Neue Deutsche Biographie, Red. Franz Menges, Bernhard Ebneth, Stefan Jordan, Maria Schimke, Reginne Sontag. 21:535-537. Berlin: Duncker \& Humboldt.

Bötticher, Georg. 1890. Kleinmichel Julius, Herrn Dietchens Erzählungen und andere Dialekthumoresken. Leipzig: Franz Karrer.

Bötticher, Georg. 1890. Schnurrige Kerle! Humoresken von G. Bötticher. Leipzig: Franz Karrer.

Bötticher, Georg, 1890. Schilda. Verse eines Kleinstädters. Leipzig: Franz Karrer. 
Brockhoff, Eva Maria. 1987. „Lohmeyer Julius“. W Neue Deutsche Biographie, Red. Hans Körner, Hans Jaeger, Franz Menges, Friedrich Nemec, Claus Priesner, Christoph Schwingenstein 15:133-134 Berlin: Duncker \& Humboldt.

Celius, U. B. 1882. Aus dem Zauberland! Alte Märchen. Leipzig: Verlag von Edwin Schloemp.

Dittmar, Franz und Julius Kleinmichel. 1886. Aus der Jugendzeit. Geschichte für die Kinderwelt. Leipzig: Verlag von E. Twietmeyer.

Donop v. 2016. „Rosenfelder Karl Ludwig R.“ W Deutsche Biographie Onlinefassung, Dostęp Lipiec 25, 2019. https://www.deutsche-biographie.de/ sfz76967.html.

Hoffmann, Franz. 1877. Die Großmutter im Kreise ihrer Enkel. Stuttgart: Nitzschke Verlag.

Holland, Hyacinth. 2016. „Kleinmichel, Fr. Julius“. W Deutsche Biographie - Onlinefassung. Dostęp Styczeń 1, 2019. https://www.deutsche-biographie.de/ sfz42614.html.

Jäger, Clara. 1881. Die Wunderflasche. Wie du mir, so ich dir. Zwei Schauspiele für die Jugend. Stuttgart: Kröner.

Kleinmichel, Ferdinand Julius Theodor. B. d. „Playtime”. W Blouin Art. Dostęp Styczeń 5, 2019. https://blouinartsalesindex.com/auctions/Ferdinand-Julius-Theodor-Kleinmichel-4927072/Playtime-.

Kleinmichel, Ferdinand Julius Theodor. B. d. „Alte Frau in der Kapelle“. W Blouin Art. Dostęp Styczeń 1, 2019. https://blouinartsalesindex.com/auctions/ Ferdinand-Julius-Theodor-Kleinmichel-4993665/Alte-Frau-in-der-Kapelle-.

Kleinmichel, Ferdinand Julius Theodor. 1883. „The first step”. W Blouin Art. Dostęp Styczeń 1, 2019. https://blouinartsalesindex.com/auctions/Ferdinand-Julius-Theodor-Kleinmichel-1084228/The-first-step-1883.

Kleinmichel, Julius. 1877. „1846 Rodzonne - 1892 Munich Sunday Afternoon on the Grafenberger Chausee". W Invaluable. Dostęp Styczeń 1, 2019. https:// www.invaluable.com/auction-lot/1846-rodzonne-1892-munichsunday-afternoonon-th-682-c-9d545dbbf8.

Kleinmichel, Julius. 1878. "Little girl with toothache". W Bukowskis - Arts \& business. Dostęp Styczeń 1, 2019. https://www.bukowskis.com/en/lots/797903-julius-kleinmichel-julius-kleinmichel-oil-on-canvas-signed-and-dated-leipzig-78.

Kleinmichel, Julius und Victor Blüthgen. 1881. Im Flügelkleide. Bilder \& Reime aus der Kinderwelt. Berlin: Hofmann.

Kleinmichel, Julius, Julius Lohmeyer. 1887. Mein Vaterhaus. Ein heiteres Kindertagebuch. Leipzig: Verlag von Meissner \& Buch.

Kleinmichel, Julius und Carl Röhling. 1886. Kinderwelt. 230 Lieder, Sprüche und Reime. Berlin: Grote.

Klotz, Aiga. 1990. Kinder- und Jugendliteratur in Deutschland 1840-1950, Gesamtverzeichnis der Veröffentlichungen in deutscher Sprache, 1. Stuttgart: J. B. Metzlersche Verlagsbuchhandlung. 
Klotz, Aiga. 1994. Kinder- und Jugendliteratur in Deutschland 1840-1950, Gesamtverzeichnis der Veröffentlichungen in deutscher Sprache, 3. Stuttgart: J. B. Metzlersche Verlagsbuchhandlung.

Klotz, Aiga. 1999. Kinder- und Jugendliteratur in Deutschland 1840-1950, Gesamtverzeichnis der Veröffentlichungen in deutscher Sprache, 5, Stuttgart: J. B. Metzlersche Verlagsbuchhandlung.

Koester, Hermann L. 1906. Geschichte der Deutschen Jugendliteratur in Monographien. Hamburg: Alfred Janssen.

Kramer, Ludwig. 1883. Für kleine Leute. Bilder und Reime. München: Bassermann.

Lohmeyer, Julius, Johan Trojan und Julius Kleinmichel. 1886. Kinderhumor. Leipzig: Meissner \& Buch.

Lohmeyer, Julius, Julius Kleinmichel und Schanz Frida. 1888. Eine Prinzenreise. Mitte heiteren Reimen von Frida Schanz und Julius Lohmeyer. Leipzig und New York: Baldamus.

Marlitt, Eugenie. 1899. Reichsgräfin Gisela. Leipzig: Union Deutsche Verlagsgesellschaft Stuttgart Berlin Leipzig.

Müller, Hermann Alex. 1882. Biographisches Künstler-Lexikon. Leipzig: Leipzig, Bibliographisches Institut.

Murray, Charles Augustus. 1890. Prärievogel. Stuttgart-Berlin-Leipzig, Union Deutsche Verlagsgesellschhaft.

NN. 2013. „Kate Greenaway (1846-1901)”. W The Victorian Web. Ostatnia modyfikacja Styczeń 18, 2019. http://www.victorianweb.org/art/illustration/greenaway/index.html.

Ringelnatz, Joachim, Pape, Walter. 1974. Parodie und Selbstparodie in Leben und Werk. Berlin und New York: Walter de Gruyter.

Schmid, Christoph. 1910. Hundert kurze Erzählungen. München und Oldenbourg.

Szymanowski, Wacław, 1883. Obrazków świat z dziecinnych lat: opowiadania wierszem autora „Złotej rószczki” z rycinami kolorowanemi według akwarelli J. Kleinmichla. Petersburg: Bolesław Maurycy Wolff .

Trojan, Johan und Julius Kleinmichel,. 1880. Die Welt vom Fenster aus. Breslau: C. T. Wiskott.

Wildermuth, Adelheid. 1898. Mein Bruder und ich. Stuttgart - Berlin - Leipzig: Union Deutsche Verlagsgesellschaft.

Wiercińska, Janina. 1986. Sztuka i Książka. Warszawa. Państwowe Wydawnictwo Naukowe.

Zarych, Elżbieta. 2015. Ilustracje dla dzieci. W Encyklopedia dzieciństwa. Dostęp Czerwiec 27, 2019. http://encyklopediadziecinstwa.pl/index.php/llustracje_dla_dzieci. 


\section{Janusz Bonczkowski}

Archiwum Państwowe w Toruniu, Polska

e-mail: jbonczkowski@torun.ap.gov.pl

folia

ORCID ID: 0000-0003-1033-2802

Dr Janusz Bonczkowski jest pracownikiem Archiwum Państwowego w Toruniu, absolwentem kierunku Historia - specjalność Archiwistyka na Wydziale Nauk Historycznych Uniwersytetu Mikołaja Kopernika w Toruniu oraz Studium Podyplomowego w zakresie Programowania i Zastosowania Komputerów. W 2017 r. uzyskał stopień doktora nauk humanistycznych za dysertację: „Analiza dyplomatyczno-źródłoznawcza księgi czynszowej miasta Grudziądza z lat 1502-1516 wraz z jej edycją źródłową". Jego zainteresowania koncentrują się wokół historii miasta Grudziądza i innych małych miast Prus Królewskich w okresie staropolskim, a także edycji źródeł. Jest członkiem Stowarzyszenia Archiwistów Polskich i Towarzystwa Naukowego w Toruniu.

\section{Fryderyk Juliusz Kleinmichel (Friedrich Julius Kleinmichel) - malarz rodzajowy i ilustrator (1846-1892)}

DOI: http://dx.doi.org/10.12775/FT.2019.012

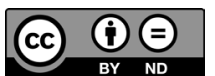

Tekst jest opublikowany na zasadach niewyłącznej licencji Creative Commons Uznanie autorstwa-Bez utworów zależnych 4.0 Międzynarodowe (CC BY-ND 4.0).

łowa kluczowe: Ludwig Richter; Kate Greenaway; Victor Blüthgen; Helen Binder, Emma Biller; Julius Lohmeyer; Johannes Trojan; Franz Dittmar; Rodzone; Lubawa; Królewiec; Düsseldorf; Lipsk; Monachium

\section{$S$}

treszczenie. Cel/Tez: tematem niniejszego artykułu jest postać Fryderyka Juliusza Kleinmichela, pochodzącego ze wsi Rodzone pod Lubawą malarza rodzajowego, ilustratora książek i czasopism. Metoda badawcza: aby przedstawić sylwetkę i twórczość Fryderyka Juliusza Kleinmichela przejrzano dostępną literaturę, XIX w. gazety i książki z jego ilustracjami a także katalogi z wystaw i aukcji jego obrazów. Wyniki/wnioski: Tematyka obrazów oraz ilustracji do książek i gazet Fryderyka Juliusza Kleinmichela dotyczyła głównie świata dziecięcego. Współpracował przede wszystkim z pisarzami i poetami literatury dziecięcej takimi jak Victor Blüthgen, Helen Binder, Emma Biller, Julius Lohmeyer, Johannes Trojan, Franz Dittmar. 


\section{Janusz Bonczkowski}

State Archives in Toruń, Poland

e-mail: jbonczkowski@torun.ap.gov.pl

ORCID ID: 0000-0003-1033-2802

Janusz Bonczkowski, $\mathrm{PhD}$, is an employee of the State Archives in Toruń, a graduate of History - specialization in Archival Sciences at the Faculty of Historical Sciences of Nicolaus Copernicus University in Toruń and Postgraduate Studies in Computer Programming and Application. In 2017 he obtained the degree of doctor of humanities for his dissertation: "Diplomatic and source analysis of the rent book of the city of Grudziądz from 1502-1516 along with its source edition". His interests focus on the history of the city of Grudziądz and other small cities of Royal Prussia in the Old Polish period; another field of scientific interest concerns editing sources. He is a member of the Association of Polish Archivists and the Scientific Society in Toruń.

\section{Friedrich Julius Kleinmichel - a genre painter and illustrator (1846-1892)}

DOI: http://dx.doi.org/10.12775/FT.2019.012

(c) $\bigodot_{\mathrm{BY}}$ The text is available under a Creative Commons Attribution-NoDerivatives 4.0
International (CC BY-ND 4.0).

eywords: Ludwig Richter; Kate Greenaway; Victor Blüthgen; Helen Binder, Emma Biller; Julius Lohmeyer; Johannes Trojan; Franz Dittmar; Rodzone; Lubawa; Konigsberg; Düsseldorf; Leipzig; Munich

ummary. Aim: The subject of this article is the figure of Friedrich Julius Kleinmichel, a genre painter, illustrator of books and magazines from the village of Rodzone near Lubawa. Research method: available literature, $19^{\text {th }}$ century newspapers and books with illustrations as well as catalogues from exhibitions and auctions of his paintings were reviewed to present the silhouette and work of Friedrich Julius Kleinmichel. Results/conclusions: The subject of paintings and illustrations for books and newspapers of Friedrich Julius Kleinmichel mainly concerned children's world. He worked mainly with writers and poets of children's literature such as Victor Blüthgen, Helen Binder, Emma Biller, Julius Lohmeyer, Johannes Trojan, Franz Dittmar. 